\title{
BMJ Open Prospective cohort protocol examining the perioperative indicators for complications and early mortality following hip fracture surgery in the frail patient
}

\author{
Louis de Jong (D) , ${ }^{1}$ Veronique van Rijckevorsel, ${ }^{1}$ Taco M A L Klem, ${ }^{2}$ \\ Martijn Kuijper, ${ }^{1}$ Gert R Roukema ${ }^{1}$
}

To cite: de Jong L, van Rijckevorsel V, Klem TMAL, et al. Prospective cohort protocol examining the perioperative indicators for complications and early mortality following hip fracture surgery in the frail patient. BMJ Open 2020;10:e038988. doi:10.1136/ bmjopen-2020-038988

- Prepublication history for this paper is available online. To view these files, please visit the journal online (http://dx.doi. org/10.1136/bmjopen-2020038988).

Received 01 April 2020

Revised 28 July 2020

Accepted 29 July 2020

Check for updates

(C) Author(s) (or their employer(s)) 2020. Re-use permitted under CC BY-NC. No commercial re-use. See rights and permissions. Published by BMJ.

${ }^{1}$ Department of Surgery, Maasstad Hospital, Rotterdam, The Netherlands

${ }^{2}$ Department of Surgery, Franciscus Gasthuis en Vlietland Hospital, Rotterdam, The Netherlands

Correspondence to

Dr Louis de Jong;

dejonglouis@hotmail.com

\section{ABSTRACT}

Introduction The primary aim is to validate earlier suggested risk factors and to find new associated risk factors for (30-day) mortality after a hip fracture in the frail population. The secondary aim is to determine the factors associated with perioperative complications. At last we want to develop and validate a more specific 30-day mortality prediction tool compared with the Nottingham Hip Fracture Score. The 30-day mortality prediction can help inform surgical risk and guide shared decisionmaking among patients, family and physicians.

Methods and analysis The study is designed as a prospective multicentre cohort study within the area of Rotterdam, the Netherlands starting from January 2018. All patients over 65 years of age, with an acute proximal hip fracture, are included. Treatment of patients will be by standard practice of care using the latest national and international guidelines. Inclusion will be continued at least until January 2021 and including at least 2500 patients. In this large cohort we hope to have sufficient strength and quality to identify risk factors of 30-day mortality and to compare them to known risk factors in literature. Moreover, we plan to develop and validate a 30-day mortality prediction tool, which identifies patients with a high probability of 30-day mortality.

Ethics and dissemination Ethical approval for this protocol was given by the Ethics Committee of the Maasstad Hospital (TWOR). Patient data are stored anonymously using the Castor data management system. No external funding is used for this study. Results will be published in peer-reviewed publications and at international conferences.

Trial registration number NL8313.

\section{INTRODUCTION}

Fractures of the hip are associated with high overall morbidity and mortality rates. Following a hip fracture, even frail patients with major comorbidities are in need for surgery, aiming for pain relief and early mobilisation. ${ }^{12}$ Complications after surgery are inevitable in this fragile population and

\section{Strengths and limitations of this study}

- Large and comprehensive prospective hip fracture database.

- The design of a prospective ongoing database, research questions related to complications and clinical outcomes can be answered.

- This study is not a randomised controlled trial focusing on specific research questions.

high incidence rates of delirium (23\%-39\%), pneumonia $(5.9 \%)$, surgical site infections $(5 \%)$ and myocardial infarction $(1.9 \%)$ have been reported. ${ }^{1-6}$ In addition to high complication rates, high mortality rates are reported after hip fracture surgery. Early postoperative mortality is particularly high, with reported 30-day mortality rates of $5.4 \%-13.3 \% .^{6-10}$ Age, comorbidities, perioperative management and postoperative complications mostly influence the risk of mortality. ${ }^{611-14}$ Determination of risk factors supports clinicians to identify patients at high risk for mortality and enables accurate preoperative risk assessment. A known high risk for mortality can support appropriate informed consent (patient and family), timing of surgery, and enable possibilities for intervention with respect to perioperative management.

Risk factors for mortality identified in previous literature are age, male gender, cognitive impairment, multiple comorbidities, diabetes, cancer, abnormal ECG, institutionalisation, functional impairment (prefracture mobility), body mass index, a high American Society of Anesthesiologists classification and infections during admission. ${ }^{121315}$

The primary aim of the present study is to validate earlier suggested risk factors and find 
new associated factors for 30-day mortality. The secondary aims are:

- To collect epidemiological data about the background and incidence of patients with a hip fracture in the Rotterdam area between 2018 and 2022.

- To analyse clinical outcomes such as in-hospital mortality, length of hospital stay and complications after surgery to gain a more complete insight in the postoperative outcome.

- To monitor the implementation and functioning of a geriatric trauma unit within orthopaedic trauma units.

- To develop a more specific 30-day mortality prediction tool compared with the Nottingham Hip Fracture Score (NHFS) for clinical usage.

\section{PATIENTS AND METHODS}

\section{Project context}

Based on the results of our previous studies in the FranciscusHospital and Maasstad Hospital in Rotterdam we found a 30-day mortality rate of $9.5 \%$ after hip hemiarthroplasty in patients with proximal femur fractures. ${ }^{16} \mathrm{In}$ that study baseline characteristics and clinical outcomes were retrospectively obtained from the hospital records. For a more specific and definitive answer to prognostic factors for 30-day mortality in our population, we will have to include patients with a proximal femur fracture in a prospective study design with all types of proximal femur fractures included (femoral neck, pertrochanteric and subtrochanteric fractures). The primary outcome is mortality within 30 days after admission. This time frame is chosen because 30 days is the common follow-up period after surgery to study the association between perioperative mortality and a hip fracture. ${ }^{10} 1718$ Mortality at 3 and 12 months after surgery will be analysed as secondary outcomes.

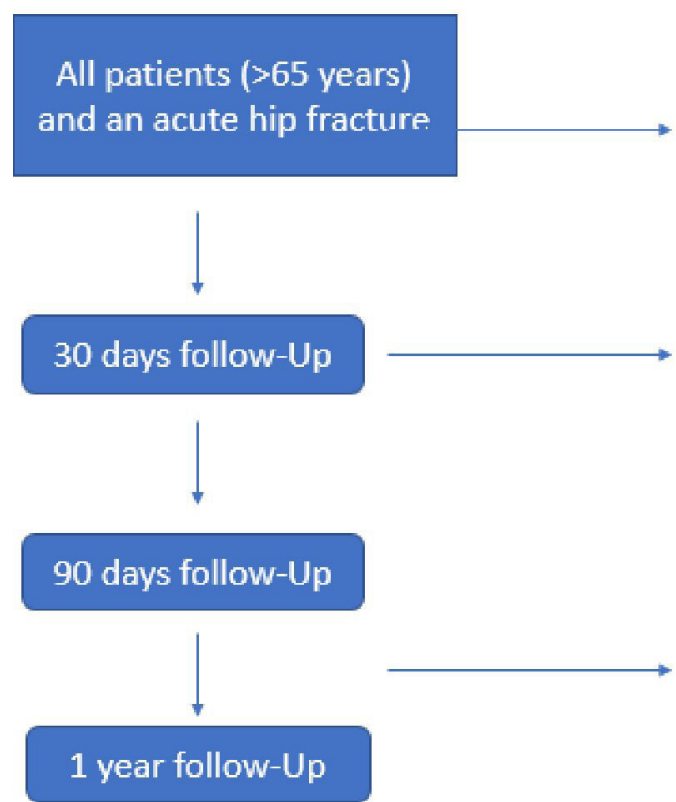

\section{Study design and population}

The study is designed as a prospective cohort study within the area of Rotterdam, the Netherlands starting from January 2018 (figure 1). All patients over 65 years of age, with an acute proximal hip fracture (intracapsular, trochanteric or subtrochanteric), are included. Excluded are patients with multitrauma injuries, pathological fractures without sufficient trauma mechanism, or patients with no understanding of the Dutch or English language. Table 1 provides an overview of the planned collection of baseline characteristics of the patients. Treatment of patients will be by standard practice of care using the latest national and international guidelines. After admission at the emergency department a pelvic $\mathrm{X}$-ray is made as soon as possible. After diagnosing a hip fracture, the patient is transferred to orthopaedic trauma department. A geriatrician is consulted prior to surgery for each patient with a hip fracture. If the patient is known with cognitive impairment or suspected of having it, the patient will be admitted to the geriatric trauma unit within the orthopaedic trauma department. Within the geriatric trauma unit a dedicated geriatric team of nurses is present to treat the patient in an environment which aims to imitate a nursing home facility as close as possible. From day 1 after surgery the patient is mobilised with physiotherapy and the patients are eating and staying in a living room during the day to facilitate a day and night rhythm. Our previous studies showed that $30 \%$ of patients were admitted from a semi-independent nursing home or nursing home facility. ${ }^{19}$ The remaining patients were in need of recovery in a nursing home facility after surgery. Therefore, from day 1 after surgery there is an inventory and application for aftercare and revalidation in a nursing home facility.

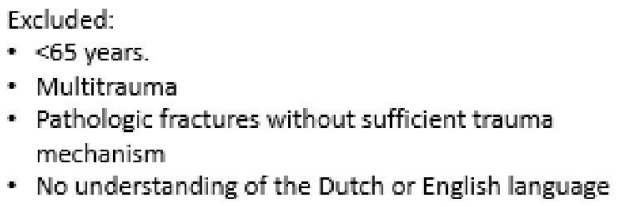

\section{Cause of death}

Mortality percentage

Factors affecting 30-day

mortality

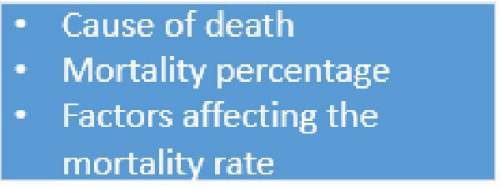


Table 1 Collection of baseline characteristics of the patients

Factor

Baseline characteristics

Age

Gender

BMI

ASA score

NHFS

Residential status

Walking aids (inside/outside)

Katz ADL index

$\begin{array}{llll}\text { Cardiac comorbidities } & \text { Rhythm anomalies } & \text { Valve insufficiency } & \text { Myocardial infarction Hypertension } \\ \text { Pulmonary comorbidities } & \text { COPD } & \\ \text { Brain comorbidities } & \text { Cerebrovascular accident } & \text { Parkinson's } \\ \text { Cognitive dysfunction } & \text { Dementia } & \text { Psychiatric disorders }\end{array}$

Malignancy

\begin{tabular}{|c|c|c|}
\hline Musculoskeletal & Osteoporosis & Previous fractures \\
\hline Kidney failure & GFR & \\
\hline Endocrine failure & Diabetes mellitus & \\
\hline
\end{tabular}

Autoimmune disease Rheumatoid arthritis

Vascular disease

Use of medication

ASA, American Society of Anesthesiologists; BMI, body mass index; COPD, chronic obstructive pulmonary disease; GFR, glomerular filtration rate (in mL/min/1.73 $\mathrm{m}^{2}$ ); Katz ADL, Katz Index of Independence in Activities of Daily Living; NHFS, Nottingham Hip Fracture Score.

\section{Mortality prediction tools}

Preoperative risk factors for mortality have been identified, ${ }^{6} 121319$ and various risk stratification tools assessing patients' risk of morbidity and mortality, such as the Physiological and Operative Severity Score for the Enumeration of Mortality and Morbidity, the Charlson Comorbidity Index, the NHFS and modifications (Almelo Hip Fracture Score), have been developed to predict 30-day mortality risk after surgery. ${ }^{80-26}$ In comparison to other models the NHFS shows the most promising results after extensive validation and with reasonable discrimination. ${ }^{2127}$ To verify whether the mortality after an intracapsular hip fracture was higher compared with the predicted mortality score according to the NHFS, a validation of the NHFS in patients with a hemiarthroplasty after an intracapsular hip fracture was performed in one of our previous studies. ${ }^{16}$ Findings suggest that for a patient with a hemiarthroplasty following an intracapsular hip fracture, there could be an underestimation for the 30-day mortality rate following the NHFS prediction model. ${ }^{16}$ In our prospective database the NHFS will be calculated and used as a benchmark model for prediction of 30-day mortality.

\section{Data analyses and statistical power}

After 3 years of inclusion there will be an estimated number of 2500 patients in the prospective database.
After completing follow-up, the primary aim is to search for factors associated with 30-day mortality. A prediction model for 30-day mortality will be fitted by logistic regression. Variable selection will be performed by applying the lasso or elastic net methodology in conjunction with 10 -fold cross-validation to prevent overfitting. To describe the characteristics of included patients, categorical variables will be presented as frequencies and percentages. Continuous variables will be presented as mean $( \pm \mathrm{SD})$. The nature of the correlation (eg, linear and quadratic) between candidate mortality predictors and outcome will be graphically assessed by locally weighted scatterplot smoothing curves. For a non-linear correlation, cut-off points are determined based on the observed distribution, clinical grounds and literature. Table 2 describes the planned collection of perioperative variables. Mortality and clinical outcome parameters such as delirium, wound infections and factors affecting length of hospital stay will be analysed. Mortality rates and differences in 30-day mortality influenced by complications and clinical outcomes will be estimated and displayed using the Kaplan-Meier estimator. Statistical analyses will be performed using Stata (StataCorp, College Station, TX, USA). All statistical tests will be two sided with a significance level of $\mathrm{p}<0.05$. 
Table 2 Planned collection of perioperative variables

\section{Factor}

\begin{tabular}{|c|c|}
\hline $\begin{array}{l}\text { 1. Preoperative } \\
\text { characteristics }\end{array}$ & 3. Treatment characteristics \\
\hline Date and time of fracture & Type and use of anaesthesia \\
\hline Date and time of admission & Type of implant \\
\hline Cause of accident & Date and time of surgery \\
\hline Location of accident & Duration of surgery \\
\hline Fracture type & Use of drain \\
\hline Fracture side & Anticoagulation \\
\hline \multirow[t]{2}{*}{ Pathological fracture } & Complications during surgery \\
\hline & $\begin{array}{l}\text { Blood loss intraoperative } \\
\text { medication }\end{array}$ \\
\hline
\end{tabular}

\section{Admission details}

\begin{tabular}{ll} 
Department & Mortality during admission \\
\hline $\begin{array}{l}\text { Involvement of geriatrician } \\
\text { Complications during }\end{array}$ & $\begin{array}{l}\text { Date ready for discharge } \\
\text { admission }\end{array}$ \\
$\begin{array}{l}\text { Clinical outcomes of } \\
\text { admission }\end{array}$ & Discharge location \\
\hline $\begin{array}{l}\text { Involvement of } \\
\text { physiotherapy }\end{array}$ & Walking aids at discharge \\
SNAQ score &
\end{tabular}

SNAQ, Short Nutritional Assessment Questionnaire.

\section{Time plan of the study}

The study is designed as a prospective cohort study within the area of Rotterdam, the Netherlands starting from January 2018. Inclusion will be continuing at least until January 2021. During this period patients will be included in at least two level II trauma hospitals in Rotterdam (the Franciscus Hospital and the Maasstad Hospital). If inclusion is started and well on time in these two hospitals the inclusion can be extended to more level II trauma centres in the region of Rotterdam. In the first two hospitals an estimated number of 900 patients per year receive surgery for their hip fracture. During the 3 years of inclusion an estimated number of at least 2700 patients will be included.

\section{Follow-up}

Each patient will have at least 6 weeks of follow-up at the outpatient clinic. In case of a fragile or cognitively impaired patient the follow-up will be performed by calling the nursing home facility. Table 3 describes the variables collected at 6 weeks and 1 year of follow-up. If a patient has died during follow-up, the cause and date of death will be noted.

\section{Patient and public involvement}

Patients and/or the public were not involved in the development of the study protocol.
Table 3 Planned collection of follow-up variables at 6 weeks and 1 year

\begin{tabular}{ll}
\hline Factor & \\
\hline Follow-up at $\mathbf{6}$ weeks & Follow-up at $\mathbf{1}$ year \\
\hline Date of follow-up & Date of follow-up \\
\hline Alive or deceased & Alive or deceased \\
Complications & Complications \\
Mobility level & Mobility level \\
Residential status & Residential status \\
Osteoporosis screening & \\
\hline Katz ADL index & \\
\hline
\end{tabular}

Katz ADL, Katz Index of Independence in Activities of Daily Living.

\section{DISCUSSION}

In the present paper we cover the design, outcome measures and timeline of the Rotterdam hip fracture study Factors affecting mortality and morbidty rates after hip fracture surgery, FAMMI study). After completion of 3 years of inclusion with at least 30 days of follow-up the prospective database will include an estimated 2700 patients, with a minimum of 2500 included patients. The primary objective of the study is to validate earlier suggested risk factors and to identify new associated factors for 30-day mortality after hip fracture surgery. Using penalised logistic regression using the lasso or elastic net procedure, factors will be selected and combined to develop a 30-day mortality prediction model. Moreover, epidemiological data on the background and incidence of patients with hip fracture in the Rotterdam area will be analysed and combined with clinical outcomes such as: in-hospital mortality, length of hospital stay and complications after surgery to gain a more complete insight in the postoperative outcome.

\section{Preoperative care}

If a patient is admitted from a nursing home with the suspicion of a hip fracture, the pelvic X-ray is made at the emergency department as soon as possible. In case of a hip fracture the patient will be admitted to the hospital at the orthopaedic trauma department, and in case of cognitive dysfunction or multiple comorbidities the patient will be admitted at the geriatric trauma unit within the orthopaedic trauma department. On admission the patient's medication and comorbidities are verified and if necessary other specialists are consulted prior to surgery. Anticoagulation use is verified and inhibitors are administered if necessary. In our earlier study between 2011 and 2016 the mean time to surgery was 27 hours, and $93 \%$ (851 patients) had surgery within 48 hours on admission. ${ }^{6}$ The time from arrival at the emergency department until admission at the department should be as short as possible in order to reduce waiting time for surgery. By fast-tracking patients with hip fracture straight to the orthopaedic ward, Eriksson et al were able to decrease the mean time from arrival to start of surgery 
and the majority of these patients underwent surgery within 24 hours. ${ }^{28}$ Pincus $e t ~ a l^{18}$ investigated in over 42000 patients whether 30-day mortality was higher if the operation was delayed for more than 24 hours. Indeed, they showed that an increased risk of mortality was present if more than 24 hours between admission and surgery had expired. ${ }^{29}$ In most national guidelines and literature a time to surgery of less than 48 hours is advised to reduce postoperative mortality. In the recent publication of the randomised controlled trial of the HIP ATTACK investigators there was no benefit in accelerated surgery within 6 hours after diagnosis compared with 24 hours (standard care group 10-42 hours). ${ }^{30}$ We want to measure and validate the optimal time to surgery within the 48 hours before the risk of postoperative complications increases. Moreover, we wish to study whether the time to surgery should be strictly within 24 hours on admission or can be extended to 48 hours. Besides the time to surgery, timing of surgery will be studied. For example, whether surgery during evenings or weekends will cause more complications such as delirium compared with planned surgery during the day.

\section{Perioperative care}

As mentioned before, dedicated geriatric trauma units within hospitals are needed to provide the surrounding care during an admission of a hip fracture and to reduce postoperative complications. ${ }^{31}$ From 2015 onwards, a geriatrician was consulted prior to surgery as standard practice of care for each patient ( $>70$ years) presenting with hip fracture. The consultation of a geriatrician became standard practice of care because most fragile patients with hip fracture have multiple physical comorbidities in combination with cognitive impairment. Multiple factors lead to a delirium percentage of $26 \%$ in our population after hip fracture surgery. ${ }^{19}$ In our prospective study we will investigate whether admission at the geriatric trauma unit combined with the consultation of geriatrician prior to surgery will reduce the percentage of complications, more specifically the incidence and length of delirium.

\section{Postoperative care}

Early mobilisation after surgery with a physiotherapist is needed to reduce postoperative complications such a pneumonia and delirium. As mentioned before, from day 1 after surgery an application is made for further revalidation outside the hospital in order to reduce the length of in-hospital stay. During admission at the geriatric trauma unit an activity mentor is present who will try to facilitate a normal day schedule as much as possible for all patients with hip fracture during their admission, including eating, crafting and playing games together in the living room.

\section{Strengths and limitations}

To our knowledge the present study will be a large and comprehensive prospective database compared with known literature studying factors effecting 30-day mortality after proximal femur fracture. With the design of a prospective ongoing database, more research questions related to complications and clinical outcomes can be answered. However, since its design is a prospective cohort, differential loss to follow-up can introduce bias and absence on data on potential confounding factors can lead to false conclusions. The follow-up for patients with a hip fracture is in general short since a high percentage of patients will die within a few years after surgery. Moreover, this study is no randomised controlled trial focusing on specific research questions. The level of evidence is therefore lower for these specific questions. However, our study could provide new clinical information necessary for the start of randomised controlled trials.

\section{Trial status}

When this manuscript was submitted, recruitment has started.

\section{ETHICS AND DISSEMINATION}

Ethical approval for this protocol was given by the Ethics Committee of the Maasstad Hospital (TWOR). Patients will receive standard practice of care after their hip fracture. Because of the high percentage of cognitive dysfunction and no changes are made to the standard practice of care, the local ethics committee (TWOR Rotterdam) decided that patients' consent to review their medical records is not required. Patient data are stored anonymously using the Castor data management system, and all the protocols are to be conducted in compliance with the Declaration of Helsinki. No external funding is used for this study.

Acknowledgements The orthopaedic trauma surgeons of the Franciscus Hospital (GB Schmidt, NMR Soesman, AGJ van Marle, JM van Buijtenen, F van Beek and TMALK) and Maasstad Hospital (GRR, CH van der Vlies, NWL Schep, BI Cleffken, J Vermeulen) are highly acknowledged for their ongoing effort to reduce time to surgery and to improve clinical outcomes in patients with hip fracture. Moreover, the staff of the orthopaedic trauma departments and the staff of the geriatric trauma units in the Franciscus Hospital and Maasstad Hospital are highly acknowledged for their hard work to provide a high standard of care for the fragile population of patients with hip fracture.

Contributors LdJ acted as trial principal investigator. VvR and LdJ enrolled patients and collected data. MK performed statistical analysis of the trial data. LdJ drafted the manuscript. GRR, TMALK, VvR and MK critically revised the manuscript. All authors have read and approved the final manuscript.

Funding The authors have not declared a specific grant for this research from any funding agency in the public, commercial or not-for-profit sectors.

Competing interests None declared.

Patient consent for publication Not required.

Provenance and peer review Not commissioned; externally peer reviewed.

Open access This is an open access article distributed in accordance with the Creative Commons Attribution Non Commercial (CC BY-NC 4.0) license, which permits others to distribute, remix, adapt, build upon this work non-commercially, and license their derivative works on different terms, provided the original work is properly cited, appropriate credit is given, any changes made indicated, and the use is non-commercial. See: http://creativecommons.org/licenses/by-nc/4.0/.

ORCID iD

Louis de Jong http://orcid.org/0000-0002-4085-6861 


\section{REFERENCES}

1 Jameson SS, Khan SK, Baker P, et al. A national analysis of complications following hemiarthroplasty for hip fracture in older patients. QJM 2012;105:455-60.

2 Nichols Cl, Vose JG, Nunley RM. Clinical outcomes and 90-day costs following hemiarthroplasty or total hip arthroplasty for hip fracture. $J$ Arthroplasty 2017;32:S128-34.

3 Gleason LJ, Schmitt EM, Kosar CM, et al. Effect of delirium and other major complications on outcomes after elective surgery in older adults. JAMA Surg 2015;150:1134-40.

4 Krogseth M, Wyller TB, Engedal K, et al. Delirium is a risk factor for institutionalization and functional decline in older hip fracture patients. J Psychosom Res 2014;76:68-74.

5 Yang $\mathrm{Y}$, Zhao X, Dong T, et al. Risk factors for postoperative delirium following hip fracture repair in elderly patients: a systematic review and meta-analysis. Aging Clin Exp Res 2017;29:115-26.

6 de Jong L, Klem TMAL, Kuijper TM, et al. Factors affecting the rate of surgical site infection in patients after hemiarthroplasty of the hip following a fracture of the neck of the femur. Bone Joint $J$ 2017;99B:1088-94

7 Dubljanin Raspopovic E, Markovic Denic L, Marinkovic J, et al. Early mortality after hip fracture: what matters? Psychogeriatrics 2015;15:95-101.

8 Nijmeijer WS, Folbert EC, Vermeer M, et al. Prediction of early mortality following hip fracture surgery in frail elderly: the Almelo hip fracture score (AHFS). Injury 2016;47:2138-43.

9 Wiles MD, Moran CG, Sahota O, et al. Nottingham hip fracture score as a predictor of one year mortality in patients undergoing surgical repair of fractured neck of femur. Br J Anaesth 2011;106:501-4.

10 Tsang C, Boulton C, Burgon V, et al. Predicting 30-day mortality after hip fracture surgery: evaluation of the National hip fracture database case-mix adjustment model. Bone Joint Res 2017;6:550-6.

11 Khan MA, Hossain FS, Ahmed I, et al. Predictors of early mortality after hip fracture surgery. Int Orthop 2013;37:2119-24.

12 Hu F, Jiang C, Shen J, et al. Preoperative predictors for mortality following hip fracture surgery: a systematic review and metaanalysis. Injury 2012;43:676-85.

13 Smith T, Pelpola K, Ball M, et al. Pre-operative indicators for mortality following hip fracture surgery: a systematic review and metaanalysis. Age Ageing 2014;43:464-71.

14 de Jong L, Klem TMAL, Kuijper TM, et al. The minimally invasive anterolateral approach versus the traditional anterolateral approach (Watson-Jones) for hip hemiarthroplasty after a femoral neck fracture: an analysis of clinical outcomes. Int Orthop 2018;42:1943-8.

15 Starcević S, Suljagić V, Stamenković D, et al. In-hospital mortality analysis in patients with proximal femoral fracture operatively treated by hip arthroplasty procedure. Vojnosanit Pregl 2016;73:251-5.

16 de Jong L, Mal Klem T, Kuijper TM, et al. Validation of the Nottingham hip fracture score (NHFS) to predict 30-day mortality in patients with an intracapsular hip fracture. Orthop Traumatol Surg Res 2019;105:485-9.

17 Marufu TC, White SM, Griffiths R, et al. Prediction of 30-day mortality after hip fracture surgery by the Nottingham hip fracture score and the surgical outcome risk tool. Anaesthesia 2016;71:515-21.

18 Pincus D, Ravi B, Wasserstein D, et al. Association between wait time and 30-day mortality in adults undergoing hip fracture surgery. JAMA 2017;318:1994-2003.

19 de Jong L, van Rijckevorsel VAJIM, Raats JW, et al. Delirium after hip hemiarthroplasty for proximal femoral fractures in elderly patients: risk factors and clinical outcomes. Clin Interv Aging 2019;14:427-35.

20 Ramanathan TS, Moppett IK, Wenn R, et al. Possum scoring for patients with fractured neck of femur. Br J Anaesth 2005;94:430-3.

21 Maxwell MJ, Moran CG, Moppett IK. Development and validation of a preoperative scoring system to predict 30 day mortality in patients undergoing hip fracture surgery. Br J Anaesth 2008;101:511-7.

22 Moppett IK, Parker M, Griffiths R, et al. Nottingham hip fracture score: longitudinal and multi-assessment. $\mathrm{Br} J$ Anaesth 2012;109:546-50.

23 Moppett IK, Wiles MD, Moran CG, et al. The Nottingham hip fracture score as a predictor of early discharge following fractured neck of femur. Age Ageing 2012;41:322-6.

24 Charlson ME, Pompei P, Ales KL, et al. A new method of classifying prognostic comorbidity in longitudinal studies: development and validation. J Chronic Dis 1987;40:373-83.

25 Mohamed K, Copeland GP, Boot DA, et al. An assessment of the POSSUM system in orthopaedic surgery. J Bone Joint Surg Br 2002;84:735-9.

26 Jonsson $\mathrm{MH}$, Bentzer P, Turkiewicz A, et al. Accuracy of the physiological and operative severity score for the enUmeration of mortality and morbidity score and the Nottingham risk score in hip fracture patients in Sweden - a prospective observational study. Acta Anaesthesiol Scand 2018;62:1057-63.

27 Karres J, Heesakkers NA, Ultee JM, et al. Predicting 30-day mortality following hip fracture surgery: evaluation of six risk prediction models. Injury 2015;46:371-7.

28 Eriksson M, Kelly-Pettersson P, Stark A, et al. 'Straight to bed' for hip-fracture patients: a prospective observational cohort study of two fast-track systems in 415 hips. Injury 2012;43:2126-31.

29 Pincus D, Wasserstein D, Ravi B, et al. Reporting and evaluating wait times for urgent hip fracture surgery in Ontario, Canada. CMAJ 2018;190:E702-9.

30 HIP ATTACK Investigators. Accelerated surgery versus standard care in hip fracture (hip attack): an international, randomised, controlled trial. Lancet 2020;395:698-708.

31 Kalmet PHS, Koc BB, Hemmes B, et al. Effectiveness of a multidisciplinary clinical pathway for elderly patients with hip fracture: a multicenter comparative cohort study. Geriatr Orthop Surg Rehabil 2016;7:81-5. 